\title{
Workflow for 3D geovisualization of the data obtained with the use of Unmanned Aerial Vehicle in Augmented Reality
}

\author{
Łukasz Halik $^{\mathrm{a}, * \text {, Maciej Smaczyński }{ }^{\text {a }} \text {, Beata Medyńska-Gulij }}{ }^{\mathrm{a}}$ \\ ${ }^{a}$ Affiliation for all authors: Department of Cartography and Geomatics, Adam Mickiewicz University, Poznan, Poland \\ email author 1: lukasz.halik@amu.edu.pl,email author2: maciej.smaczynski@amu.edu.pl, email author3: bmg@amu.edu.pl \\ * Corresponding author
}

Keywords: Augmented Reality, Unmanned Aerial Vehicles, Digital Terrain Model, AR, UAV, DTM

\section{Abstract:}

The attempt to work out the geomatic workflow of transforming low-level aerial imagery obtained with unmanned aerial vehicles (UAV) into a digital terrain model (DTM) and implementing the 3D model into the augmented reality (AR) system constitutes the main problem discussed in this article. The authors suggest the following workflow demonstrated in Fig. 1.

The series of pictures obtained by means of UAV equipped with a HD camera was the source of data to be worked out in the final stage of the geovisualization. The series was then processed and a few point clouds were isolated from it, being later used for generating test 3D models.

The practical aim of the research conducted was to work out, on the basis of the UAV pictures, the 3D geovisualization in the AR system that would depict the heap of the natural aggregate of irregular shape. The subsequent aim was to verify the accuracy of the produced 3D model. The object of the study was a natural aggregate heap of irregular shape and denivelations up to 11 meters.

Based on the obtained photos, three point clouds (varying in the level of detail) were generated for the 20000 -metersquare area. The several-centimeter differences observed between the control points in the field and the ones from the model might corroborate the usefulness of the described algorithm for creating large-scale DTMs for engineering purposes. The method of transformation of pictures into the point cloud that was subsequently transformed into 3D models was employed in the research, resulting in the scheme depicting the technological sequence of the creation of 3D geovisualization worked out in the AR system. The geovisualization can be viewed thanks to a specially worked out mobile application for smartphones.

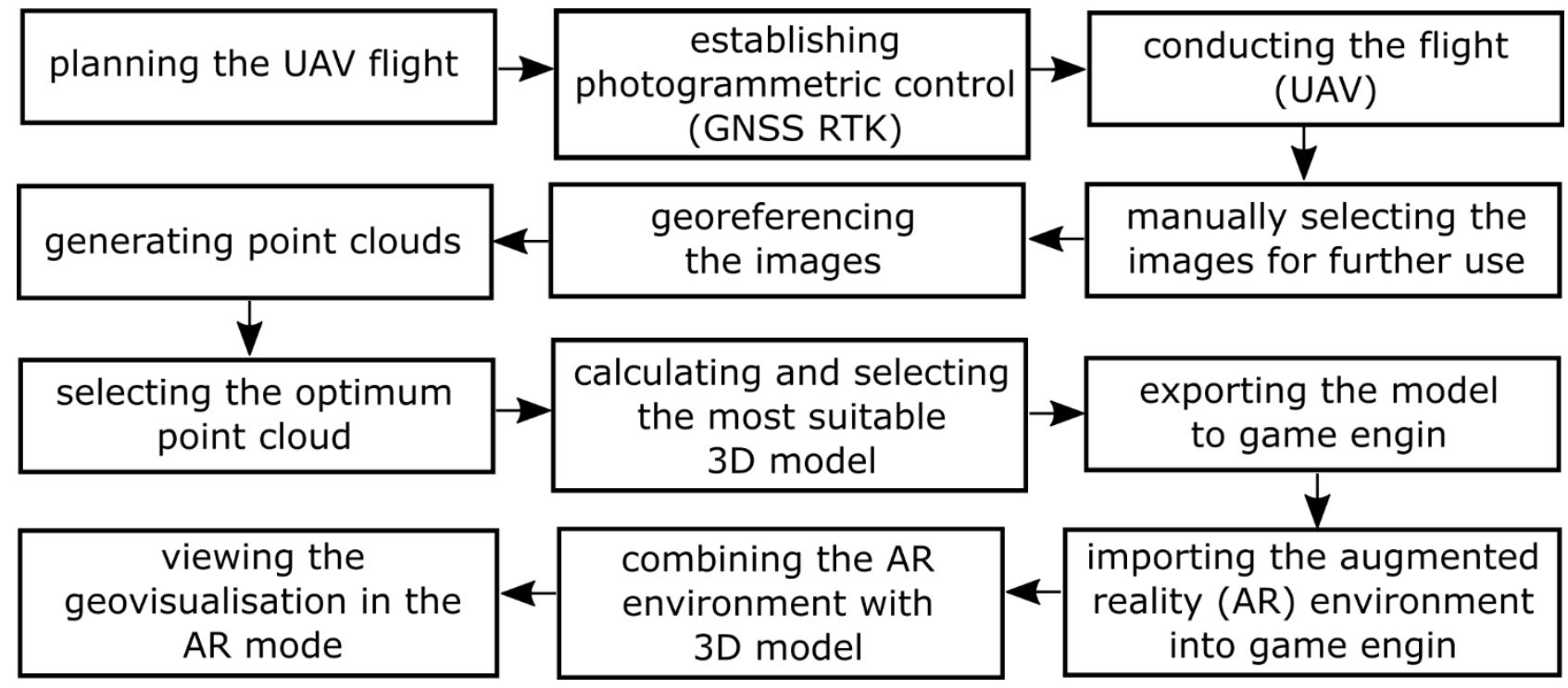

Figure 1. Schematic workflow. 